Apidologie, 1978, 9 (1), 41-55.

\title{
ÉTUDE BIOMÉTRIQUE DE DEUX POPULATIONS D'ABEILLES CÉVENOLES (1)
}

\author{
Biometrische Untersuchung an zwei Cevennen-Bienenvölkern
}

\author{
J. M. CORNUET, J. FRESNAYE, P. LAVIE \\ avec la collaboration technique de \\ J. BLANC, Sylvie HANOUT* et Catherine MARY-LAFARGUE* \\ Station Expérimentale d'Apiculture, I.N.R.A. \\ F-84140 Montfavet \\ *Institut technique de l'Apiculture, \\ F-91440 Bures sur Yvette
}

\section{SUMMARY}

BIOMETRICAL STUDY OF TWO HONEYBEE POPULATIONS

FROM THE CEVENNES

Thanks to improvements which were proposed in a previous paper (CORNUET and al, 1975), it was possible to obtain a perfect discrimination of populations from different ecotypes (120 km apart) and a partial one with populations of the same ecotype $(8 \mathrm{~km}$ apart). This last one is essentially due to the divergence of 2 of the 6 measured characters : the "hairs" and the " $A$ " of the cubital index.

Different hypothesis able to explain this fact are examinated among which was kept up the one of a partial genetical isolation promoting the differential action of the selection and (or) the genetical drift.

\section{RÉSUMÉ}

Grâce à des améliorations proposées dans une publication précédente (CORNuET et al., 1975), il a été possible de discriminer parfaitement des populations d'écotypes différents (distantes de $120 \mathrm{~km}$ ) et partiellement des populations d'un même écotype (distantes de $8 \mathrm{~km}$ ). Cette dernière discrimination repose surtout sur la divergence de 2 des 6 caractères mesurés : la " pilosité " et le " $\mathrm{A}$ " de l'index cubital.

Différentes hypothèses susceptibles d'expliquer ce fait sont examinées, parmi lesquelles a été retenue celle d'un isolement génétique partiel favorisant l'action différentielle de la sélection et (ou) de la dérive génétique.

(1) Étude réalisée avec l'aide financière de l'Institut technique de l'Apiculture dans le cadre d'un programme de recherches sur les populations d'abeilles de France et sur leur sélection. 


\section{INTRODUCTION}

Deux études précédentes (TOMASSONE et FRESNAYE, 1971, CoRnuET et al., 1975) ont montré la possibilité de discriminer morphologiquement des populations régionales d'abeilles domestiques au sein d'une même race, en l'occurrence la race noire de France (Apis mellifica mellifica). Ces résultats confirmaient, sur des populations différentes, ceux obtenus par Lous et LeFÈvre (1971). Cette discrimination morphologique à l'aide de méthodes biométriques s'ajoute ainsi à la distinction d'entités subraciales mise en évidence par LouveAux (1966) à l'aide de travaux portant sur des caractères d'adaptation de la ruche aux conditions de miellées locales.

Afin d'accéder à une meilleure connaissance des populations françaises d'abeilles domestiques et d'estimer leur variabilité au sein de la même race, il a été décidé d'entreprendre une caractérisation morphologique des différents écotypes existants. Le récent travail de MALLET (1976) s'inscrit dans ce programme et a eu pour objet la biométrie de colonies landaises. Le nôtre porte principalement sur la région des Cévennes. Pour nous permettre de détecter une éventuelle différenciation au sein du même écotype et évaluer par la même occasion les limites de nos méthodes statistiques, nous avons prélevé des échantillons de deux groupes de colonies cévenoles distants de 8 kilomètres. Nous y avons adjoint quelques colonies du Mont Ventoux considérées comme appartenant à un écotype différent pour servir de référence par rapport aux études précédentes de discrimination d'écotypes.

\section{MATÉRIEL ET MÉTHODES}

\section{A. - Origine géographique et mode de prélèvement des abeilles}

Les abeilles cévenoles proviennent de la vallée de la Mimente, limitée au nord par la barrière du massif du Mont Bougès, au sud par le plan de Fontmort, à l'ouest par une zone rocheuse vers Florac, ces trois secteurs étant à peu près dépourvus de ruches. A l'est, la crête du col de Jalcreste sert de limite au partage des eaux entre la Méditerranée et l'Atlantique. Cette vallée relativement bien isolée représente une zone qui compte un millier de colonies d'abeilles toutes situées à moins de $960 \mathrm{~m}$ d'altitude. Il est intéressant de préciser d'autre part que 750 de ces colonies sont logées dans des ruches fixes, fabriquées dans des troncs de châtaignier évidés (BonNET, 1973). Ce type d'apiculture primitive fait que les introductions d'abeilles de races étrangères ont été rarissimes et d'autre part dans cette région relativement inacessible et moyennement mellifère, il n'y a pratiquement pas de transhumance de ruchers. On peut encore signaler que sur les 100 ruchers recensés, 67 étaient plus ou moins bien entretenus et 33 complètement abandonnés. Les petites vallées sont séparées par un relief très accentué, avec des éperons rocheux atteignant souvent des altitudes de 1200 à $1300 \mathrm{~m}$ (voir carte fig. 1). Le climat est rigoureux en hiver, les jours de gel atteignent 100 à 120 par an et il pleut 60 jours ( 1600 à $2500 \mathrm{~mm}$ ). La flore mellifère naturelle est la plus importante avec dominance du châtaignier, des prairies naturelles et des bruyères Erica cinerea (TouRN, L.) mais plus fréquemment Calluna vulgaris (SALISBuRY, L.). Cet ensemble de conditions fait que les ruches de ces localités représentent des populations d'abeilles relativement pures, adaptées à certaines conditions écologiques et non hybridées avec des races géographiques étrangères.

En Cévennes les prélèvements d'abeilles ont été effectués sur 64 ruches, dans trois ruchers et deux localités. 
Les deux premiers ruchers sont situés au nord du hameau de Mijavols (commune de Saint-Juliend'Arpaon) à deux cent mètres l'un de l'autre et ils appartiennent au même propriétaire. L'emplacement se trouve à $930 \mathrm{~m}$ d'altitude et le nombre total de colonies d'abeilles était de 35 (dont 12 ruches à cadres mobiles). Nous avons prélevé les abeilles dans 9 ruches au rucher $n^{\circ} 1$ et dans 26 ruches au rucher $n^{\circ} 2$.

Le troisième rucher est situé à la Borne (commune de Saint-Privat-de-Vallongue) dans la Haute Vallée de la Mimente à $850 \mathrm{~m}$ d'altitude et bien abrité des vents d'Ouest. Ce rucher est composé exclusivement de ruches fixes, environ 100 troncs d'arbres dont une quarantaine est peuplée. Il a été prélevé des abeilles dans 29 ruches à ce rucher $\mathrm{n}^{\circ} 3$ qui est distant de 8 kilomètres à vol d'oiseau des ruchers $\mathrm{n}^{\circ} 1$ et 2 .

Sur la carte (fig. 1) on remarquera également la position de l'emplacement du rucher " réserve " de l'I.N.R.A. créé en 1973 aux Crozes Bas (commune de Cassagnas) et qui a servi aux premières mesures sur l'abeille cévenole (LAvie et Fresnaye, 1973).

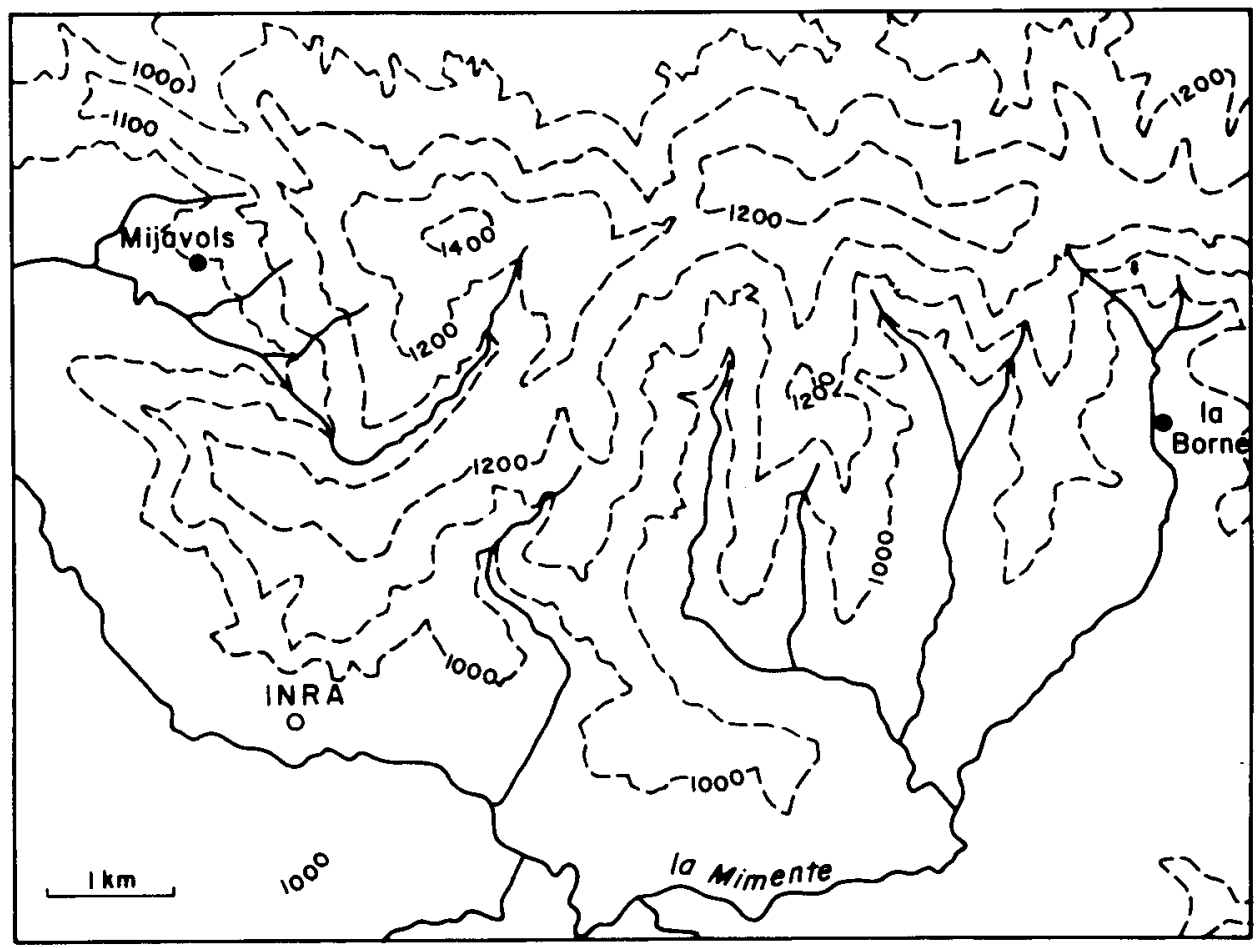

Fig. 1. - Carte géographique pour la localisation des prélèvements.

Авв. 1. - Geographischer Lageplan der Untersuchungsorte.

$\mathrm{Au}$ Ventoux, les prélèvements témoins ont été effectués de la même manière sur 14 colonies d'abeilles dans un de nos ruchers « réserve * I.N.R.A. en juillet 1975 (rucher $n^{\circ} 4$ ). Ce rucher avait été créé avec des abeilles de la localité en 1967. Les ruches se trouvent à une altitude de $1100 \mathrm{~m}$ et la flore mellifère est totalement différente de celle présente autour de nos 3 ruchers cévenols. La flore mellifère du Ventoux est constituée principalement par les lavandes sauvages (Lavandula vera (DC) et Lavandula latifolia (LC); par contre les Éricacées en sont pratiquement absentes. 


\section{B. - Caractères biométriques et méthodes de mesures}

Les analyses biométriques comportent les six caractères préconisés par divers spécialistes (GoETzE, 1963, RUTTNER, 1963, 1968) et que nous utilisons nous-mêmes depuis de nombreuses années (FRESNAYE, 1965, TOMASSONE et Fresnaye, 1971, CoRnuet et al., 1975). Les mesures sont effectuées sous loupe binoculaire selon les méthodes décrites par ailleurs de façon détaillée (FRESNAYE, 1974) et concernent :

1) La coloration mesurée sur le $2^{e}$ tergite abdominal au grossissement $\times 12$. Il s'agit de la largeur des taches ou de la bande jaune qui peut s'étendre de 0,00 à $2,40 \mathrm{~mm}$.

2) La longueur des poils sur le $5^{e}$ tergite abdominal mesurée au grossissement $\times 50$.

3) La largeur du tomentum sur le $4^{\text {e }}$ tergite abdominal mesurée au grossissement $\times 12$.

4) La longueur de la langue mesurée au grossissement $\times 16$ après avoir coupé et retourné la tête de l'abeille. La glosse est mise en extension à l'aide de pinces à dissection.

5 et 6) Les composantes $A$ et $B$ de l'index cubital mesuré à l'aide du dispositif de Ruttner au grossissement $\times 40$.

\section{C. - Méthodes statistiques}

Chaque colonie est représentée par un échantillon de 30 abeilles et nous disposons des valeurs des 6 caractères mesurés pour chaque abeille de l'échantillon. Il est donc possible de caractériser une colonie par la moyenne et la variance pour chacune des variables biométriques et par la matrice des corrélations des variables prises 2 à 2 .

Pour rechercher une éventuelle discrimination entre les divers groupes de colonies, nous avons utilisé un programme d'analyse discriminante (UNFALT) décrit par TOMASSCNE et FRESNAYE (1971) et CORNUET et al. (1975). Mais l'utilisation en a été différente puisque la colonie est maintenant considérée comme "population " et non plus comme a individu " dans l'analyse discriminante.

\section{RÉSULTATS}

\section{A. - Caractéristiques biométriques des colonies cévenoles}

Les figures 2 à 7 représentent les histogrammes des colonies pour chacun des six caractères en distinguant les contributions respectives des deux populations. Les distributions de fréquence des caractères " pilosité ", " tomentum ", "langue ", et " B " sont approximativement unimodales. Pour le caractère " $\mathrm{A}$ », les modes distincts des deux populations aboutissent à une distribution globale bimodale. Le cas de la "couleur " apparaît plus délicat : si la distribution globale est d'apparence unimodale, la population 1 semble avoir 2 modes et il est difficile d'en distinguer un dans la population 2 . Mais la faiblesse des effectifs des classes nous engage à rester très prudents dans ces affirmations.

Le tableau 1 fournit les intervalles de confiance à $5 \%$ des moyennes des deux populations pour chaque caractère mesuré, ainsi que la réponse aux tests d'égalité de ces moyennes (test $t$ de Student). Il apparait que 2 des 6 variables (" pilosité " et " $A$ ") présentent des moyennes significativement différentes (probabilité inférieure à 0,01 ) au sein des deux populations. Pour le caractère " $\mathrm{A}$ ", cela confirme l'observation précédente du caractère bimodal de la distribution. Comme la différence de pilosité est plus inattendue au regard de l'histogramme, nous avons effectué le test $U$ de Mann et Whitney d'application beaucoup moins restrictive que le test de Student. Néanmoins, les 


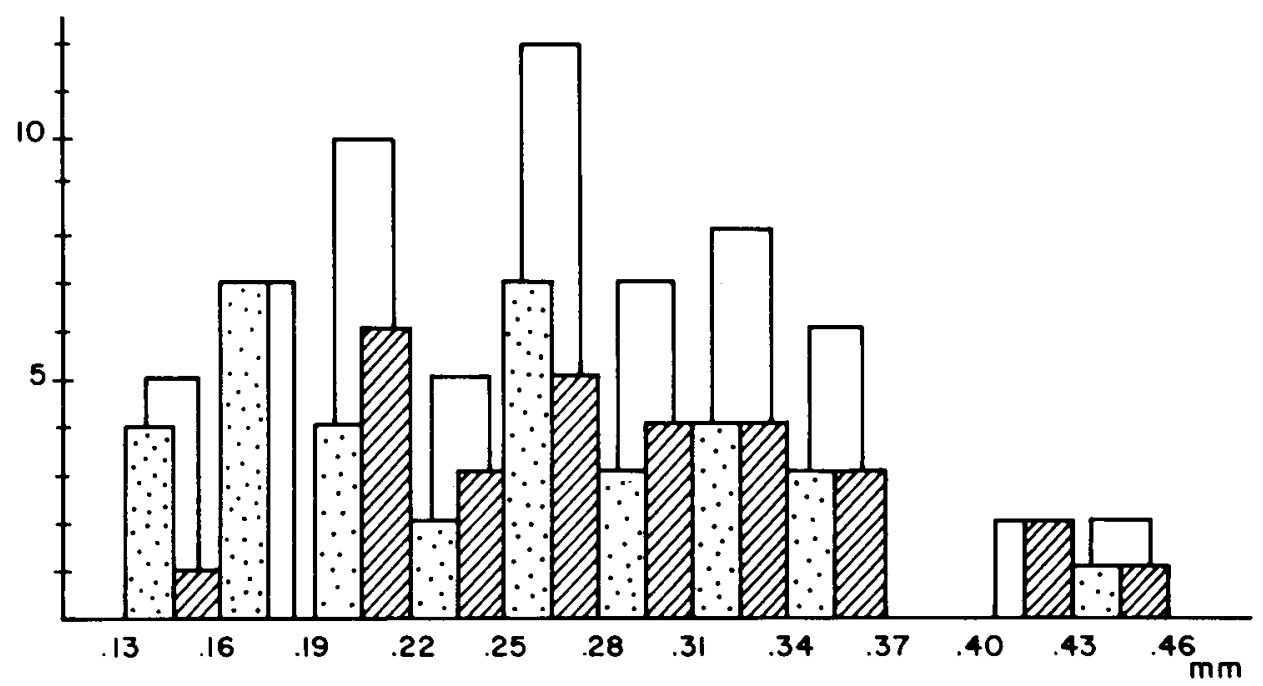

FIG. 2. - Histogramme du caractère a couleur ".

AвB. 2. - Histogramm des Merkmals " Farbe».

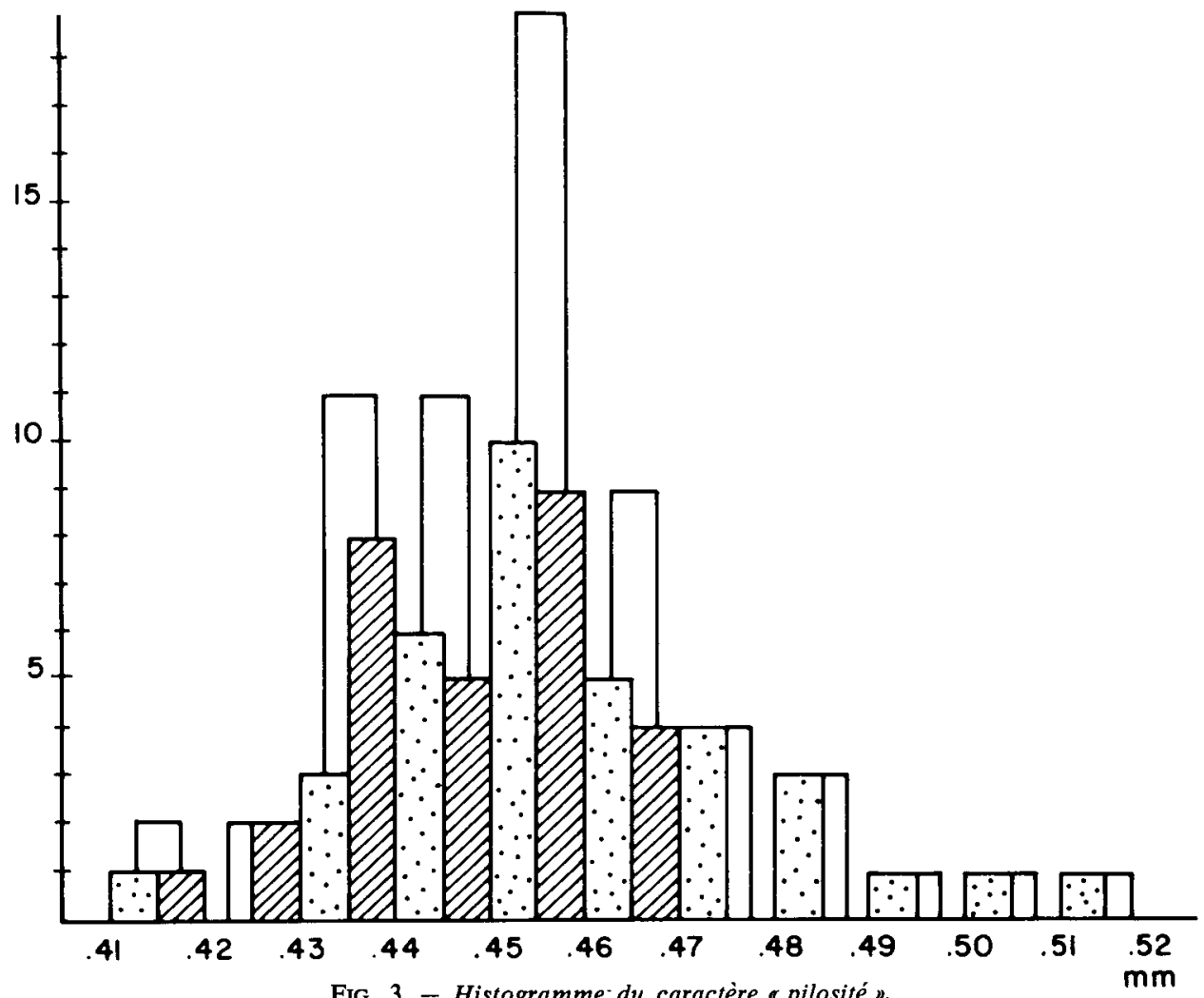

FIG. 3. - Histogramme-du caractère " pilosité 》.

ABB. 3. - Histogramm des Merkmals " Behaarung ». 


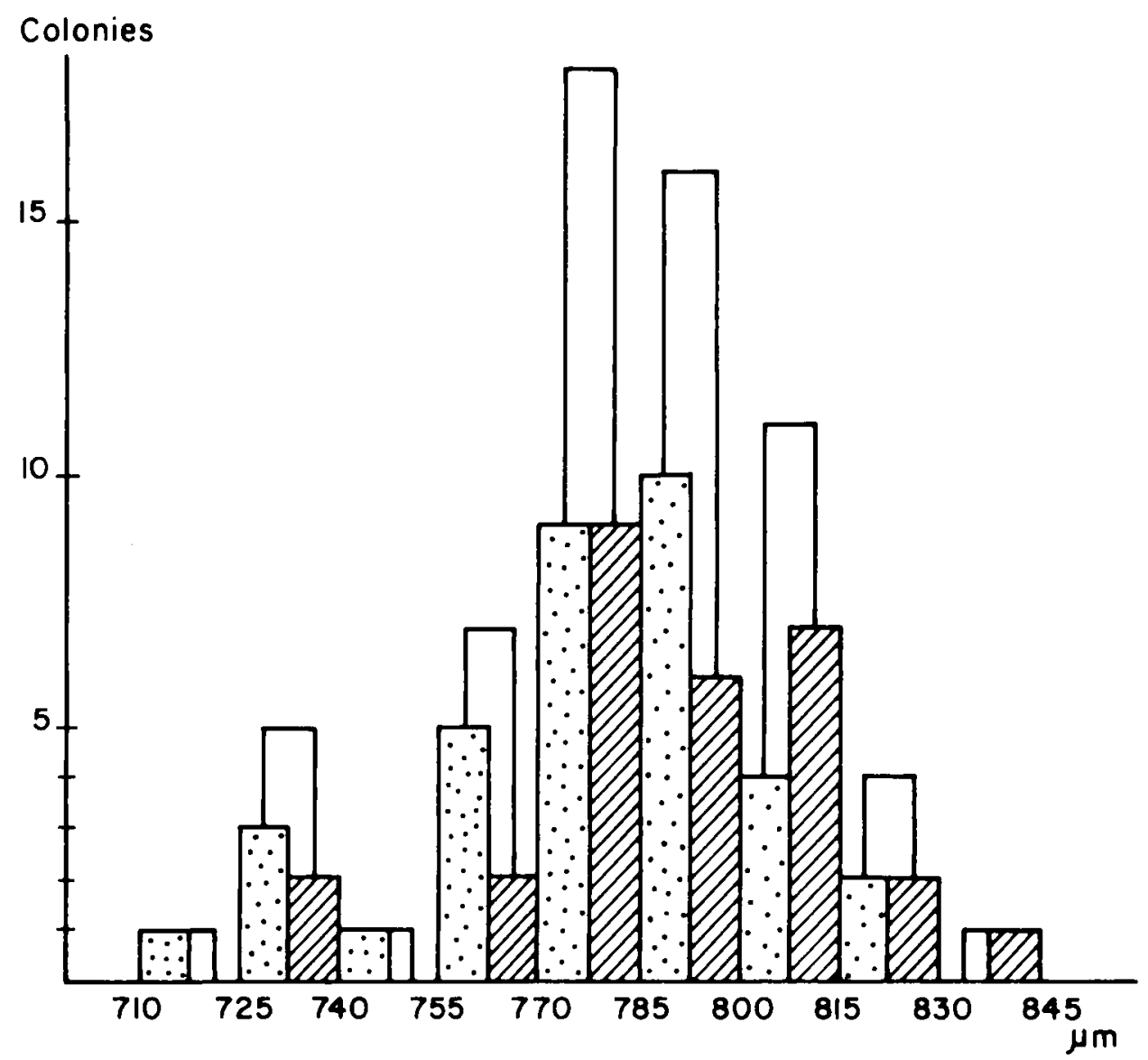

FIG. 4. - Histogramme du caractère "tomentum 》.

Авв. 4. - Histogramm des Merkmals a Filzbinde ". 


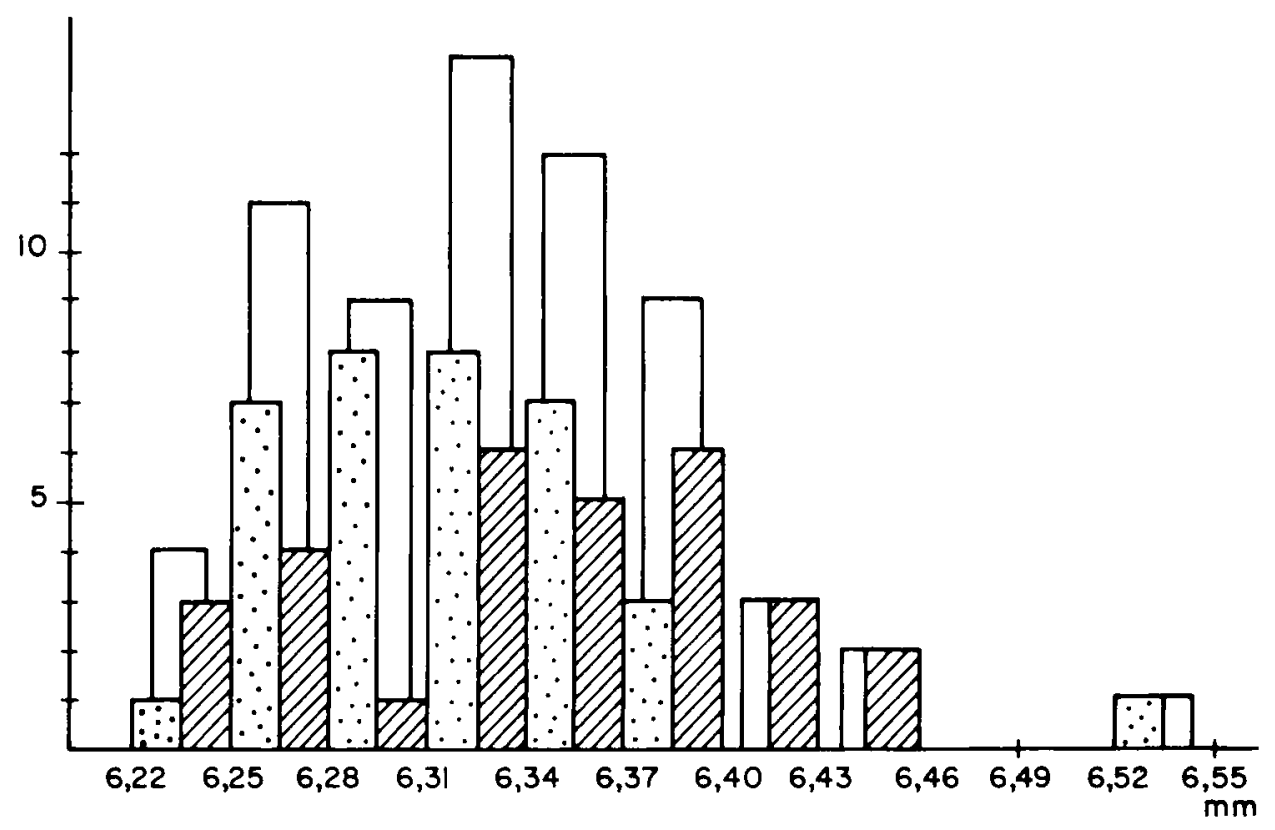

FIG. 5. - Histogramme du caractère "langue \#.

AвB. 5. - Histogramm des Merkmals "Zunge ».

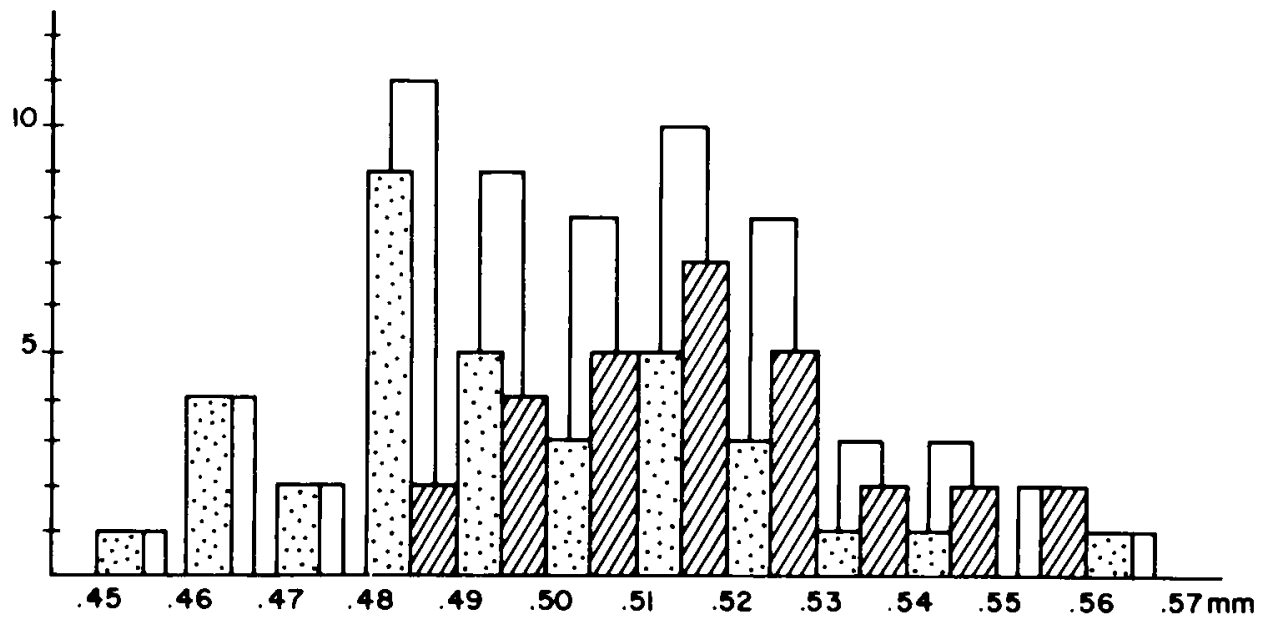

FIG. 6. - Histogramme du caractère " $A$ ע.

Авв. 6. - Histogramm des Merkmals a Abschnitt a» des Cubital - Indexes $a / b$. 


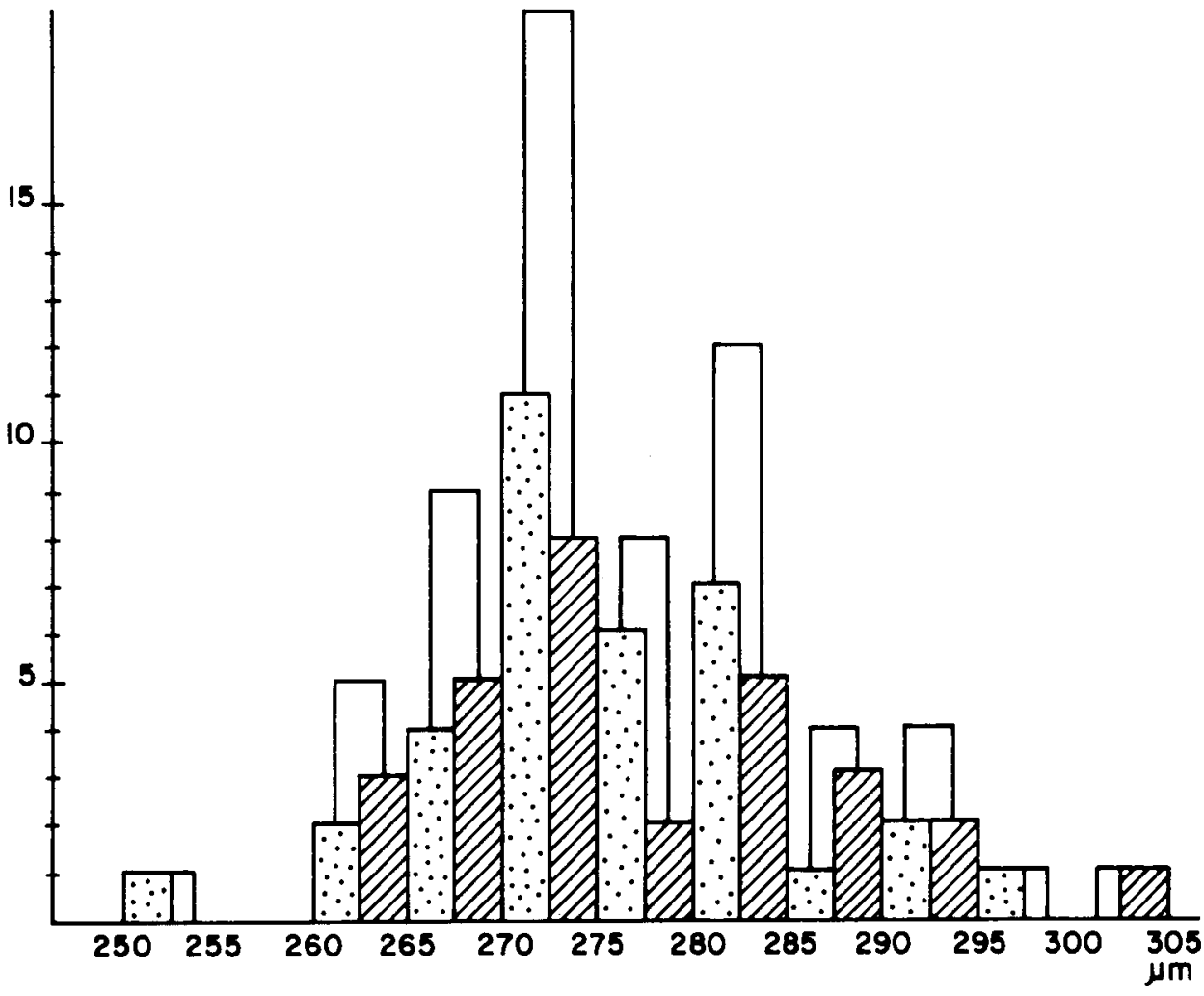

FIGURE 7. - Histogramme du caractère a $B$ ».

Aвв. 7. - Histogramm des Merkmals a Abschnitt b des Cubital - Indexes $a / b$.

Pour les figures 2, 3, 4, 5, 6, 7 : en pointillé: localité 1

en hachuré : localité 2

en blanc: cumul des 2 localités

$Z u$ den Abbildungen 2, 3, 4, 5, 6, $7:$ Punktiert $=$ Ort 1

Schraffiert $=$ Ort 2

Weiss $=$ Kumulierung beider Orte 
TABL. 1. - Intervalles de confiance à $5 \%$ et test d'égalité des moyennes des deux groupes de colonies cévenoles (toutes les données sont en millimètres). TAB. 1. - $5 \%$-Vertrauens-Intervalle und Übereinstimmungs-Test der Mittelwerte beider Gruppen von Cevennen-Völkern (Alle Angaben in $\mathrm{mm}$ ).

\begin{tabular}{|l|c|c|c|}
\hline \multicolumn{1}{|c|}{$\begin{array}{c}\text { Caractère } \\
\text { Merkmal }\end{array}$} & $\begin{array}{c}\text { Localité 1 } \\
(35 \text { colonies }) \\
\text { Ort 1 }\end{array}$ & $\begin{array}{c}\text { Localité 2 } \\
(29 \text { colonies }) \\
\text { Ort } 2\end{array}$ & $\begin{array}{c}\text { Résultat du test } \\
\text { Untersuchungsergebnis }\end{array}$ \\
\hline $\begin{array}{l}\text { Couleur } \\
\text { Farbe } \\
\text { Pilosite } \\
\text { Behaarung }\end{array}$ & $0,2493 \pm 0,0244$ & $0,2826 \pm 0,0276$ & non significatif \\
$\begin{array}{l}\text { Tomentum } \\
\text { Filzbinde } \\
\begin{array}{l}\text { Langue } \\
\text { Zunge } \\
\text { A } \\
\text { Abschnitt a }\end{array}\end{array}$ & $0,4598 \pm 0,0071$ & $0,4445 \pm 0,0053$ & P $<0,01$ \\
$\begin{array}{l}\text { B } \\
\text { Abschnitt b }\end{array}$ & $0,7798 \pm 0,0083$ & $0,7897 \pm 0,0088$ & non significatif \\
\hline
\end{tabular}

deux tests conduisent ici au même résultat : la probabilité que les deux échantillons proviennent d'une même population est inférieure à $1 \%$.

Le tableau 2 présente les moyennes et écarts-types de l'ensemble des colonies cévenoles échantillonnées.

TABL. 2. - Moyennes et écarts-types de l'écotype cévenol (toutes les données sont en millimètres).

TAB. 2. - Mittelwerte und Streuung des Cevennen-Ökotyps (Alle Angaben in $\mathrm{mm}$ )

\begin{tabular}{|l|c|c|c|c|c|c|}
\hline & $\begin{array}{c}\text { Couleur } \\
\text { Farbe }\end{array}$ & $\begin{array}{c}\text { Pilosité } \\
\text { Behaarung }\end{array}$ & $\begin{array}{c}\text { Tomentum } \\
\text { Filzbinde }\end{array}$ & $\begin{array}{c}\text { Langue } \\
\text { Zunge }\end{array}$ & $\begin{array}{c}\text { A } \\
\text { a }\end{array}$ & $\begin{array}{c}\text { B } \\
\text { b }\end{array}$ \\
\hline $\begin{array}{l}\text { Moyenne } \\
\begin{array}{l}\text { Mittelwert } \\
\text { Ecart-type } \\
\text { Streuung }\end{array}\end{array}$ & 0,264 & 0,453 & 0,784 & 6,329 & 0,506 & 0,276 \\
\hline
\end{tabular}

\section{B. - Discrimination de populations}

Nous avons effectué deux analyses discriminantes : la première en présence de 14 colonies du Mont Ventoux et la seconde avec seulement les 64 colonies cévenoles.

\section{1) Colonies cévenoles et colonies du Mont Ventoux}

Le plan discriminant $Z_{1} Z_{2}$ représentant $60,7 \%$ de la variabilité totale montre une séparation très nette entre les colonies des deux écotypes selon l'axe $Z_{1}$ (figure 8). Si l'on 


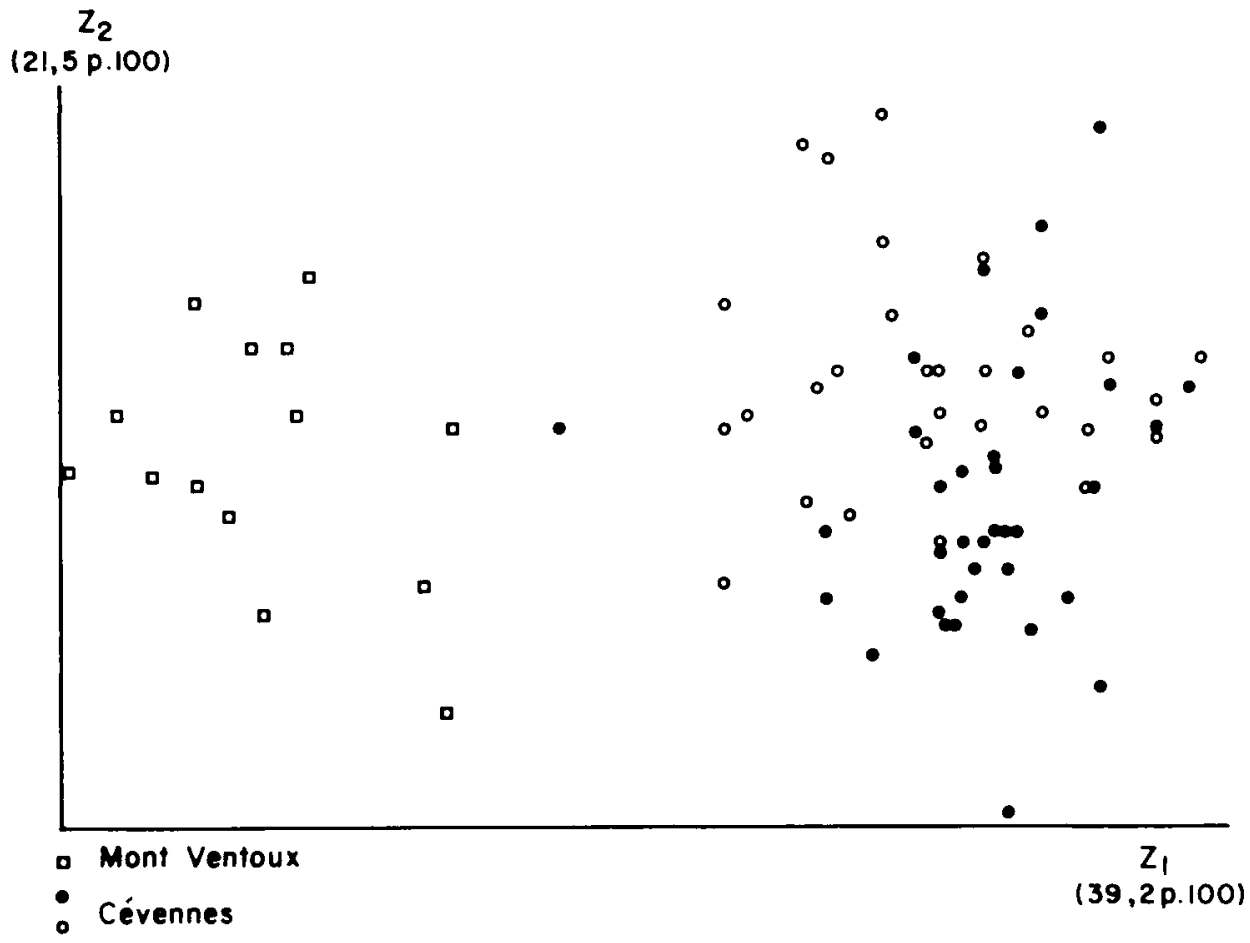

Fig. 8. - Projection des points-colonies sur le plan factoriel 1-2. Aвв. 8. - Übertragung der Punkte $=$ Völker auf den Faktor-Plan 1-2.

consulte les composantes du premier vecteur propre (tableau 3), on constate que la principale contribution vient du caractère " langue ". Ceci n'a rien d'étonnant car il apparaît au vu des données de base que toutes les colonies du Mont Ventoux ont une longueur moyenne de la langue supérieure à la valeur maximale des colonies cévenoles. Ce résultat confirme les observations préliminaires effectuées sur des abeilles cévenoles du même secteur par LAvie et Fresnaye (1973).

De plus, ont peut remarquer une certaine discrimination parmi les populations des Cévennes, cette fois selon les deux axes $Z_{1}$ et $Z_{2}$ (figure 8). La composante majeure du deuxième vecteur propre (tableau 3) correspond au caractère " $A$ », ce qui n'est pas surprenant puisqu'il a été établi au paragraphe précédent qu'il s'agit là du caractère le plus discriminant pour les deux populations cévenoles. Le troisième axe $Z_{3}(13,8 \%)$ contribue lui aussi à discriminer les colonies des Cévennes et une assez bonne discrimination de ces deux groupes s'observe dans le plan $Z_{2} Z_{3}$ (figure 9).

2) Colonies cévenoles seules

Encouragés par les résultats qui précèdent, nous espérions obtenir une amélioration en supprimant dans l'analyse discriminante les colonies du Mont Ventoux. Force 
TABL. 3. - Composantes des trois premiers vecteurs propres en fonction des variables de départ.

TAB. 3. - Komponenten der drei ersten Eigen-Vektoren in Abhängigkeit von den Ausgangs-Variabeln

\begin{tabular}{|c|c|c|c|}
\hline & $\begin{array}{l}\text { Vecteur propre } 1 \\
\text { Eigenvektor } 1\end{array}$ & $\begin{array}{c}\text { Vecteur propre } 2 \\
\text { Eigenvektor } 2\end{array}$ & $\begin{array}{c}\text { Vecteur propre } 3 \\
\text { Eigenvektor } 3\end{array}$ \\
\hline $\begin{array}{l}\text { Couleur } \\
\text { Farbe }\end{array}$ & 0,0467 & 0,0675 & $-0,5145$ \\
\hline $\begin{array}{l}\text { Pilosité } \\
\text { Behaarung }\end{array}$ & 0,1857 & $-0,2283$ & 0,6229 \\
\hline $\begin{array}{l}\text { Tomentum } \\
\text { Filzbinde }\end{array}$ & 0,0971 & $-0,0151$ & $-0,4182$ \\
\hline $\begin{array}{l}\text { Langue } \\
\text { Zunge }\end{array}$ & $-0,9769$ & $-0,0187$ & $-0,0197$ \\
\hline $\begin{array}{c}\mathrm{A} \\
\mathrm{a}\end{array}$ & 0,0300 & 1,0032 & 0,1431 \\
\hline $\begin{array}{l}B \\
b\end{array}$ & $-0,0897$ & 0,3209 & 0,4672 \\
\hline
\end{tabular}

nous est de constater que la séparation des populations cévenoles ainsi obtenue n'est pas meilleure mais d'une qualité équivalente. Il nous parait donc inutile de présenter le détail de ces résultats.

\section{DISCUSSION}

Pour caractériser morphologiquement l'écotype cévenol, nous aurions pu prélever des échantillons répartis sur l'ensemble de la région. Cela nous aurait vraisemblablement donné une image plus représentative de la biométrie de l'abeille cévenole. Mais nous n'aurions pas pu ainsi distinguer une quelconque différenciation au sein de cet écotype car comment décider à ce niveau taxonomique entre une variabilité due à l'échantillonnage et une variation représentative d'un fait biologique? Le meilleur moyen pour diminuer l'importance de la variance d'échantillonnage étant d'augmenter l'effectif de l'échantillon (aux dépens du nombre d'échantillons), nous avons décidé de ne prendre que deux échantillons d'une trentaine de colonies chacun. Cela a permis de détecter une légère différenciation portant sur au moins deux caractères morphologiques : la " pilosité " et la " $\mathbf{A}$ " de l'index cubital. Cette différenciation, certes incomplète, apparait nettement sur le plan discriminant $Z_{2} Z_{3}$ (figure 9), puisqu'une droite de ce plan permet de classer sans erreur près de $80 \%$ (50 sur 64$)$ des colonies.

Quelles peuvent être les causes d'une telle différenciation? On peut tout d'abord avancer que les différences de milieu existant entre ces deux localités conduisent à des expressions différentes de génômes semblables. Cette hypothèse est difficile à soutenir pour au moins deux raisons. La première est qu'en apparence, les conditions de milieu 


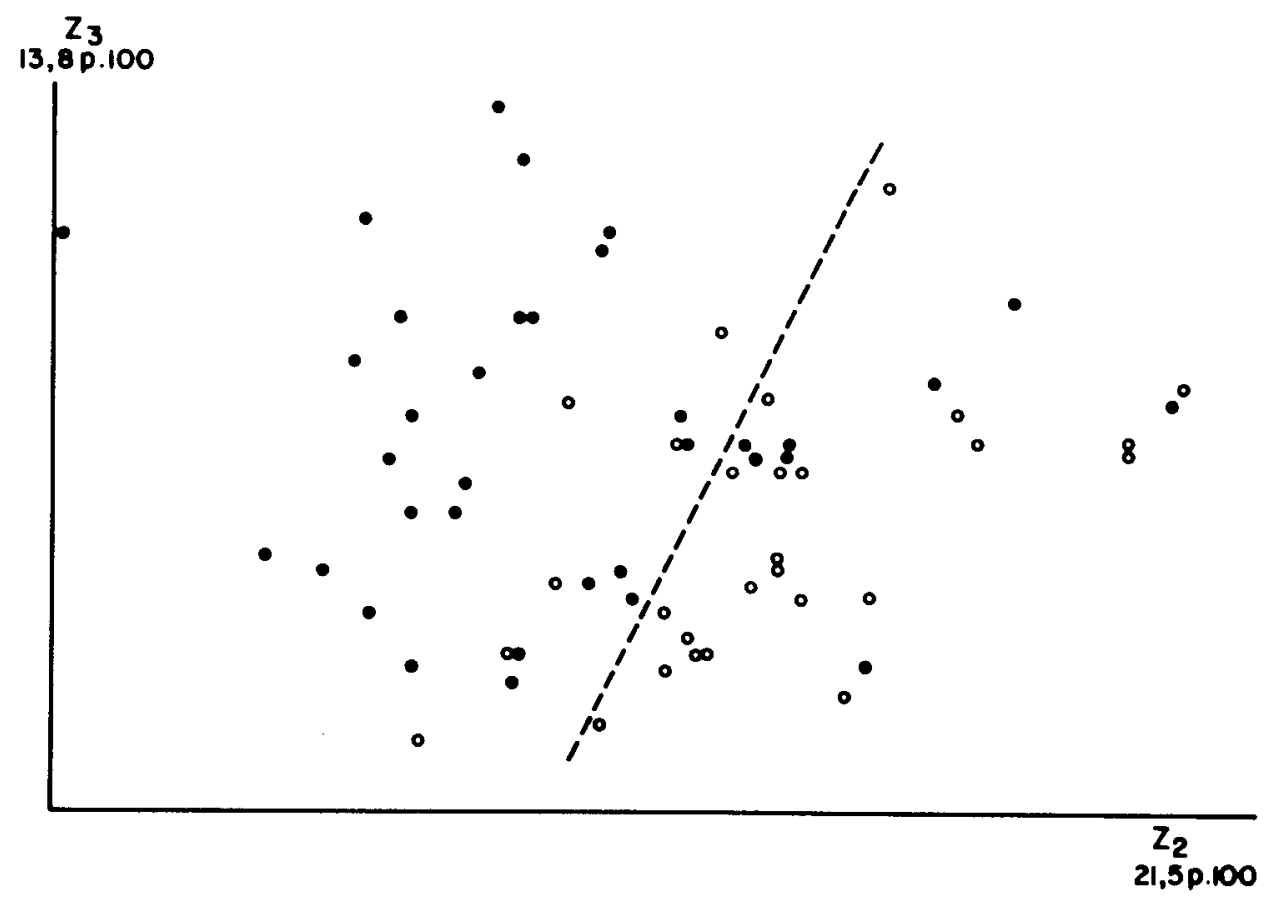

Fig. 9. - Projection des points-colonies sur le plan factoriel 2-3.

(les colonies du Mont Ventoux ne sont pas représentées).

Aвв. 9. - Übertragung der Punkte = Völker auf den Faktor-Plan 2-3.

(Die Mont Ventoux-Völker sind nicht berücksichtigt).

Pour les figures 8 et 9 : carrés blancs: Mont Ventoux

ronds noirs: localité 1 des Cévennes

ronds blancs: localité 2 des Cévennes

Zu den Abbildungen 8 und 9: Weisse Quadrate $=$ Mont Ventoux

Schwarze Kreise = Ort 1 in den Cevennen

Weisse Kreise $=$ Ort 2 in den Cevennen

semblent assez identiques dans les deux localités : mêmes conditions climatiques, même végétation, même cycle biologique annuel. La seconde raison tient à la nature des caractères mesurés. Il s'agit de caractères morphologiques à forte héritabilité (ROBERTS, 1961), donc peu sensibles aux effets du milieu.

L'autre cause possible de cette différenciation est de nature génétique : les deux populations diffèrent dans certaines fréquences géniques. Ceci implique un isolement génétique partiel permettant à la sélection et à la dérive d'opérer des évolutions génétiques divergentes. Toutefois, cette divergence reste à un niveau assez bas dans la mesure où les pressions de sélection doivent être assez voisines et où surtout les échanges de gènes ne sont pas totalement empêchés. 
Pour étayer cette hypothèse de différence génétique entre les deux populations, il conviendrait d'utiliser des méthodes permettant de connaitre de façon non ambiguë le génotype des abeilles échantillonnées. Cela est notamment possible par électrophorèse enzymatique. Cette technique a été déjà employée chez l'abeille (MESTRINer, 1969, BRÜCKNER, 1974, Sylvester, 1976) et a permis l'étude génétique d'une population insulaire (Cornuet et TORREgrossa, 1977, Cornuet, en préparation).

Revenant à la technique biométrique utilisée ici, il faut noter qu'avec six caractères seulement, nous avons pu discriminer partiellement des populations très proches et parfaitement écotypes de la même race. Ce résultat supérieur à celui de 1975 (CORNUET, et al.) rend compte de l'amélioration apportée par le changement du niveau de " population " au sens de l'analyse discriminante, changement que nous avons préconisé à la fin de cet article.

Sur l'ensemble des caractères utilisés en biométrie de l'abeille, notre choix de six caractères s'avère bon dans cette analyse puisque, dans les populations étudiées, ils apportent des informations statistiquement indépendantes (cf. les matrices de corrélations intra et inter (tabl. 4)) d'une part, et qu'ils ont été suffisants pour distinguer les deux groupes de colonies cévenoles, d'autre part.

TABL. 4. - Matrice de corrélation intra (à gauche de la diagonale) et inter (à droite de la diagonale).

TAB. 4. - Korrelationsmuster " intra (links von der Diagonalen) und * inter * (rechts von der Diagonalen).

\begin{tabular}{|c|c|c|c|c|c|c|}
\hline & $\begin{array}{l}\text { Couleur } \\
\text { Farbe }\end{array}$ & $\begin{array}{c}\text { Pilosité } \\
\text { Behaarung }\end{array}$ & $\begin{array}{c}\text { Tomentum } \\
\text { Filzbinde }\end{array}$ & $\begin{array}{l}\text { Langue } \\
\text { Zunge }\end{array}$ & $\begin{array}{l}\mathrm{A} \\
\mathrm{a}\end{array}$ & $\begin{array}{l}\text { B } \\
b\end{array}$ \\
\hline $\begin{array}{l}\text { Couleur } \\
\text { Farbe }\end{array}$ & 1 & $-0,216$ & 0,130 & 0,015 & 0,075 & 0,040 \\
\hline $\begin{array}{l}\text { Pilosité } \\
\text { Behaarung }\end{array}$ & $-0,015$ & 1 & $-0,167$ & 0,104 & $-0,106$ & $-0,041$ \\
\hline $\begin{array}{l}\text { Tomentum } \\
\text { Filzbinde }\end{array}$ & $-0,053$ & 0,049 & 1 & $-0,053$ & $-0,018$ & $-0,092$ \\
\hline $\begin{array}{l}\text { Langue } \\
\text { Zunge }\end{array}$ & $-0,020$ & 0,045 & 0,039 & 1 & 0,014 & 0,054 \\
\hline $\begin{array}{l}\mathrm{A} \\
\mathrm{a}\end{array}$ & 0,025 & 0,035 & $-0,014$ & $-0,004$ & 1 & 0,073 \\
\hline $\begin{array}{l}\text { B } \\
\text { b }\end{array}$ & $-0,019$ & 0,004 & 0,006 & 0,032 & $-0,245$ & 1 \\
\hline
\end{tabular}

\section{REMERCIEMENTS}

Nous remercions la Station de Biométrie de Jouy-en-Josas (I.N.R.A.-C.N.R.Z.) et tout particulièrement Mme Lucienne TASSENCOURT pour l'aide apportée dans les calculs statistiques. L'ordinateur utilisé est l'I.B.M. 370 du C.T.I. de l'I.N.R.A. La bibliothèque de programmes est celle du laboratoire de biométrie du C.N.R.Z. 
Nous adressons nos plus vifs remerciements à MM. M. ChAPTAL et R. BONNET grâce à qui nous avons pu effectuer les prélèvements d'abeilles dans les ruchers cévenols.

\section{RÉFÉRENCES BIBLIOGRAPHIQUES}

BonNet R., 1973. - Inventaire des ruches du versant sud du Bougès (Haute vallée de la Mimente). Note interne du Parc National des Cévennes (non publiée).

BRÜCKNER D., 1974. - Reduction of biochemical polymorphisms in Honeybees (Apis mellifica). Experientia, 30 (6), 618-619.

Cornuet J.-M., Fresnaye J., Tassencourt L., 1975. - Discrimination et classification de populations d'abeilles à partir de caractères biométriques. Apidologie, 6 (2), 145-187.

Cornuet J.-M., Torregrossa J.-P., 1977. - Variabilité génétique dans une population insulaire d'abeilles (Apis mellifica L.). XXVI ${ }^{\mathbf{e}}$ Congr. Int. Apic. Adélaïde.

CoRnuet J.-M. (En préparation). The MDH System in the honeybees (Apis mellifica L.) of Guadaloupe.

FRESNAYE J., 1965. Etude biométrique de quelques caractères morphologiques de l'abeille noire française (Apis mellifica mellifica). Ann. Abeille, 8 (4), 271-283.

Fresnaye J., 1974. Biométrie de l'abeille. Bulletin Technique Apicole O.P.I.D.A., 1 (2-3), 61370 Ste Gauburge.

GoETzE G., 1963. Die Honigbiene in natürlicher und künstlicher Zuchtauslese, Paul Parey Hamburg, $212 \mathrm{p}$.

Lavie P., et Fresnaye J., 1973. - Les particularités de l'abeille du Sud-Est de la France, in Commission Internationale de Botanique Apicole. Apidologie, 4 (2), 172-173.

Lours J., LEFĖvRE J., 1971. - Les races d'abeilles (Apis mellifica). I Détermination par l'analyse canonique. Biométrie Praximétrie, XII, 1-41.

LouveauX J., 1966. - Les modalités de l'adaptation des abeilles (Apis mellifica L.) au milieu naturel. Ann. Abeille, 9 (4) 323-350.

MALlet N., 1976. - Étude biométrique d'une population locale d'abeilles dans la grande Lande. Mémoire de fin d'études. École Nationale d'Ingénieurs des Techniques Agricoles de Bordeaux.

Mestriner M. A., 1969. - Biochemical polymorphisms in Bees (Apis mellifera ligustica). Nature, 223, 188-189.

Mestriner M. A., Contel, 1972. - The P 3 and EST loci in the honeybee (Apis mellifera). Genetics, 72, 733-738.

ROBERTS W. C., 1961. - Heterosis in the honeybee as shown by morphological characters in inbred and hybrid bees. Ann. Ent. Soc. Am., 54, 878-882.

Ruttner F., 1968. - Les races d'abeilles. Traité de biologie de l'abeille. Tome I, Masson édit. Paris, 27 44.

RUTTNER F., 1973. - Zuchttechnik und Zuchtauslese bei der Biene. Ehrenwirth Verlag, München, $138 \mathrm{p}$.

Sylvester H. A., 1976. - Allozyme variation in Honeybees (Apis mellifera L.). Ph. D. thesis University of California Davis.

Tomassone R., Fresnaye J., 1971. - Étude d'une méthode biométrique et statistique permettant la discrimination et la classification de populations d'abeilles (Apis mellifica L.). Apidologie, 2 (1), 49-65.

\section{ZUSAMMENFASSUNG}

Verschiedene Untersuchungen haben gezeigt, dass zwischen den regionalen Populationen (Ökotypen) ein-und derselben Bienenrasse eine morphologische Unterscheidung möglich ist; und man weiss, dass sechs biometrische Merkmale genügen, um einige der französischen Ökoptypen der Rasse mellifica morphologisch von einander zu unterscheiden. 
Durch die Beurteilung der gleichen Merkmale, aber unter Berücksichtigung der in einer früheren Veröffentlichung vorgeschlagenen Verbesserungen in bezug auf die Gewinnung der Bienenproben, wollten wir nachprüfen, ob es möglich wäre, eine etwaige morphologische Differenzierung innerhalb eines Ökotyps festzustellen, wobei die Grenzen der angewandten Methode durchaus in betracht gezogen wurden.

Zwei Populationen des Cevennen-Ökotyps wurden ausgewählt, die nur $8 \mathrm{~km}$ von einander entfernt aufgestellt waren (35 Bienenvölker an dem einen Ort, 29 Völker am anderen). Zusätzlich wurde eine Gruppe von 14 Völkern eines anderen Ökotyps (Mont Ventoux) hinzugenommen, um den Einfluss der abgeänderten Probenahme feststellen zu können.

Die Varianzanalyse jedes Merkmals zeigt, dass zwei von ihnen, nämlich " Behaarung " und Abschnitt " a " des Cubital-Indexes $a / b$ deutlich verschiedene Durchschnittswerte bei den beiden CevennenPopulationen aufweisen.

Die Diskriminationsanalyse trennt die Völker jedes Ökotyps sehr deutlich in bezug auf Abschnitt * a " (Abb. 8). Auf dem Faktor-Plan $\left(Z_{2}-Z_{3}\right)$ bemerkt man eine teilweise Differenzierung bei den beiden Cevennen-Populationen. Die gestrichelte Gerade in Abb. 9 führt zu einer einwandfreien Klassifizierung von 50 der insgesamt 64 Völker $=78 \%$.

Zur Beurteilung dieser Differenzierung kann man nicht einfach nur Umweltfaktoren heranziehen; denn zum einen sind die Umweltfaktoren bei einer Entfernung von $8 \mathrm{~km}$ einander sehr ähnlich (beide Bienenstände stehen in der gleichen Höhenlage ( 850 und $930 \mathrm{~m})$ ), und zum andern werden die untersuchten Merkmale von Natur aus sehr wenig durch Umweltbedingungen beeinflusst.

Man muss daher notwendigerweise einen genetischen Unterschied annehmen, der allein durch eine teilweise Isolierung der beiden Populationen erklärt werden kann. Wenn das auch a priori ziemlich überraschend scheint, so lässt sich eine solche Isolation mit Höhenlagen erklären, die hoch genug sind, um einen Austausch zwischen den beiden Orten stark einzuschränken. 